\title{
A Challenging Case of Ganglion Impar Block in a Patient with Coccygeal Deformity and Rectal Resection from Metastatic Rectal Cancer
}

\author{
Kevin Wong, DO and Brian R. Monroe, MD
}

The ganglion impar is a solitary retroperitoneal structure that marks the termination of paravertebral sympathetic chains in the presacral space and receives nociceptive inputs from pelvic organs. Chronic perineal pain (CPP) in patients with advanced cancer can be very debilitating. Ganglion impar block can ameliorate CPP and hence, improve quality of life.

Here, we describe the case of a 65-year-old woman with a deformed coccyx caused by childhood trauma and rectal resection from rectal cancer who presented with CPP that substantially affected her quality of life. She was unable to tolerate the side effects of World Health Organization Step 3 analgesics. The patient underwent ganglion impar block that was challenging due to anatomical abnormalities. There was significant immediate and long-term improvement in quality of life and disease coping in our patient after the ganglion impar block. She was able to restore physical activity, decrease the need for potent opioids, and improve sleep quality.

We conclude that a ganglion impar block can effectively treat CPP, and in palliative settings, neurolytic ablation can be considered. It is often challenging in patients with cancer due to anatomical abnormalities. Further studies are needed in patients with cancer who have CPP given the suggestion of a better quality of life after ganglion impar block.

Key words: Ganglion impar, chronic perineal pain, quality of life, cancer pain, coccyx
Chronic perineal pain (CPP) is a frequent complaint among patients with advanced stages of colorectal, prostate, and genitourinary cancer (1). It is difficult to manage because the perineum is composed of diverse anatomical structures with a complex network of sympathetic and somatic nerve fibers $(1,2)$. Patients with CPP can be effectively managed with a multidisciplinary approach through a combination of psychotherapy, antidepressants, anticonvulsants,

From: Division of Anesthesia, Geisinger Medical Center, Danville, PA Author for correspondence: Kevin Wong, DO

Address: Division of Anesthesia, Geisinger Medical Center, 100 North Academy Ave., Danville, PA 17822

E-mail: kwong@geisinger.edu analgesics, and supportive care (3). The primary goal of CPP control in patients with advanced cancer is directed towards achieving maximal restoration of functional capacity and reduction in the severity of pain (4).

Cancer pain is a complex mix of somatic, visceral, and neuropathic entities (5). All pharmacological managements have been used with varying efficacy due to the mixed nature of cancer pain (5). About $10 \%$ to $15 \%$ of patients with advanced cancer consistently fail to achieve satisfactory pain relief despite the use of World Health Organization (WHO) Step 3 analgesics (6). An opioid-based regimen may be adequate to relieve cancer pain, but it is often limited by side effects, including nausea, vomiting, pruritus, tolerance, constipation, drowsiness, respiratory de- 
pression, and hyperalgesia (5). Failure to control the chronic, unremitting nature of cancer pain can lead to substantial functional impairment and a reduced quality of life $(2,3)$. Physicians should seek interventional pain management early for patients with cancer who have CPP that is refractory to conventional medical management.

Ganglion impar, otherwise known as ganglion of Walther, is a solitary retroperitoneal structure that marks the termination of paravertebral sympathetic chains in the presacral space immediately anterior to the level of the sacrococcygeal junction $(2,3)$. It receives nociceptive inputs from visceral afferents that innervate the perineum, distal rectum, anus, distal urethra, vulva, and distal third of the vagina (7). Blockade of the ganglion impar was originally described for palliation of sympathetically mediated cancer pain involving the pelvis and perineum $(3,4)$. However, this technique is often a less preferred modality due to its technical difficulties and potential complications. Although less popular, ganglion impar block has demonstrated promising outcomes in patients with malignancy-associated perineal pain $(3,4,7)$. We report a challenging case of ganglion impar block secondary to a deformed coccyx and the absence of rectum in a patient with metastatic rectal cancer.

\section{CASE}

A 65-year-old woman with a history of coccygeal injury from childhood trauma and metastatic rectal adenocarcinoma was referred to our pain clinic for evaluation of CPP. She underwent several palliative surgeries including total abdominal hysterectomy, bilateral salpingo-oophorectomy, distal sigmoidectomy, and abdominoperineal resection (APR) with colostomy creation, followed by several rounds of palliative chemoradiotherapy. She complained of persistent sacral pain involving the posterior vaginal wall and vulvar area for the past year. The pain was described as "a red hot poker in the vagina" and "sitting on a ball." Using the visual analog scale, the patient rated the pain 10/10 in severity. The pain significantly affected her quality of life, and she could not tolerate the side effects of opioids. She was not able to sleep well and perform daily activities despite a multimodal pharmacologic approach to pain manage- ment that included acetaminophen, hydromorphone, and a fentanyl patch.

On physical examination, she was a chronically illappearing, cachetic woman with no rectum secondary to APR. The patient had an ostomy in the anterior abdominal wall, a large skin graft and pressure ulcer in the sacral area, and severe tenderness upon palpation of the sacrococcygeal area. Lateral radiographic view of the sacrum revealed a deformed coccyx with the distal coccygeal segment missing (Fig. 1). The anteroposterior view of the sacrum and coccyx showed similar findings of a deformed coccyx with an ill-defined sacrococcygeal junction (Fig. 2). This anatomical abnormality was related to her remote history of coccygeal injury from childhood trauma. A computerized tomography scan of the pelvis demonstrated significant scarring within the presacral space (Fig. 3). Despite her complicated medical history and anatomical abnormalities involving the perineum and coccyx, the patient agreed to proceed with a trial of ganglion impar block.

The patient was taken to the procedure room. A large-bore intravenous line was inserted with ease. She was placed in the lateral decubitus position with proper padding. Standard American Society of Anesthesiologists monitors were applied. After sterile prepping and draping, the perirectal region was infiltrated subcutaneously with $1 \%$ lidocaine. The initial attempt with a spinal needle inserted through the sacrococcygeal junction (Foye approach) was unsuccessful due to the significant amount of resistance from the presence of osteophytes and the anatomical distortion of the coccyx. The decision was made to block the ganglion impar using an anococcygeal instead of a transsacrococcygeal technique. A 22G, 5-inch spinal needle was curved by hand to accentuate the resistance path of the bevel. Due to the lack of anatomic markers, and significant anterior lip of the inferior coccyx, the needle was introduced caudal to the coccyx and advanced through the remnants of the anococcygeal ligament approximately one-third of the distance from the coccyx to the posterior aspect of the vagina. The needle was advanced to the presacral space under fluoroscopic guidance using the lateral view. The anteroposterior fluoroscopic view was checked intermittently to confirm that the needle had remained midline. Once fluoroscopically placed, 
the position was confirmed by injection of radiopaque dye demonstrating the classic "comma sign" (Fig. 4). No paresthesia or discomfort was reported by the patient. Injection resistance was high due to significant scarring in the presacral space and a 3 $\mathrm{mL}$ Luer Lock syringe was used rather than a "slip tip" syringe. With negative aspiration of cerebrospinal fluid and blood, a test dose of $1 \%$ lidocaine yielded considerable perineal pain relief within 2 minutes. The ganglion impar was blocked with $2 \mathrm{~mL}$ of $0.5 \%$ bupivacaine and $80 \mathrm{mg}$ of methylprednisolone acetate. The patient tolerated the procedure well without any complications. She recovered uneventfully and was discharged home in stable condition on the same day.

Upon follow-up 4 weeks later, her CPP was completely resolved, but the burning pain of

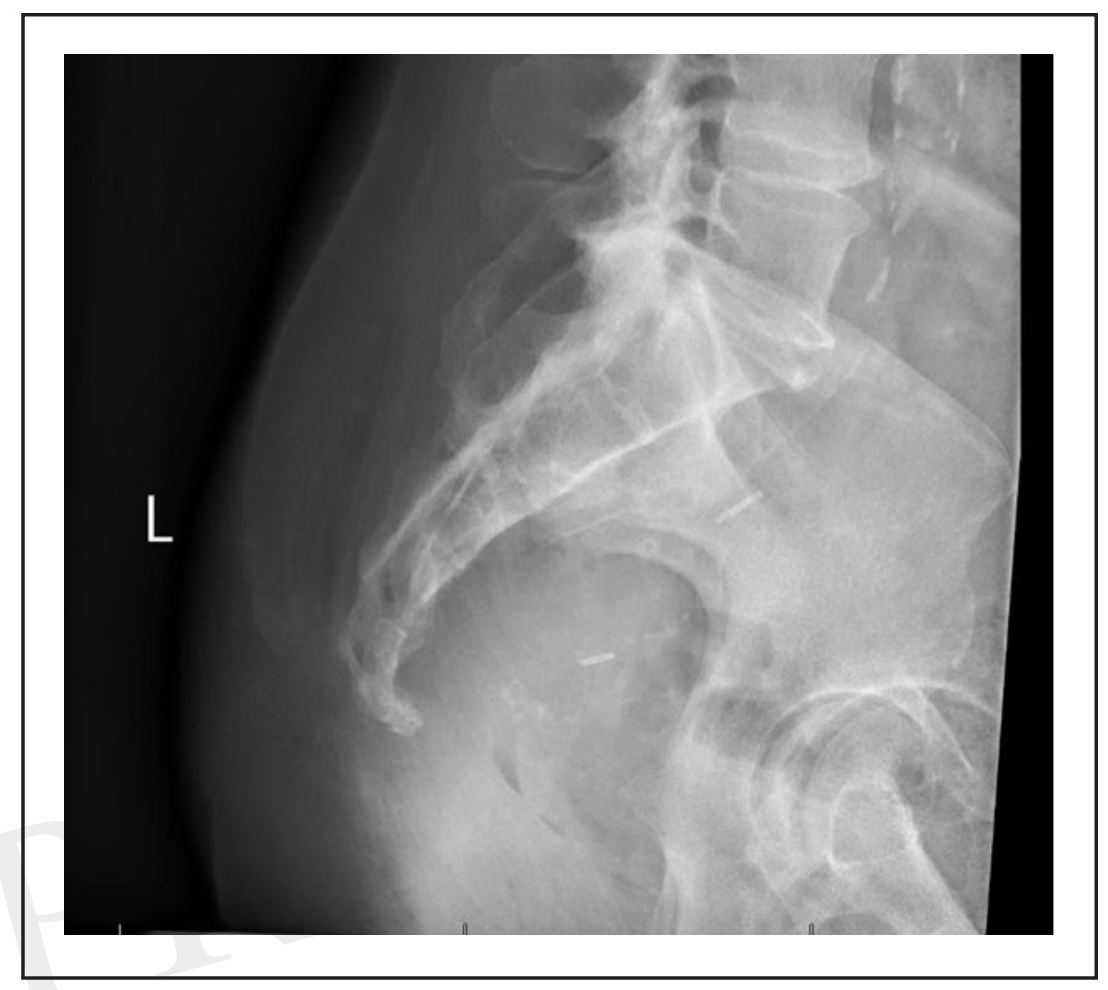

Fig. 1. Lateral radiographic view showing anatomical irregularities of the coccyx. her posterior vaginal wall and vulvar area returned at one week after the ganglion impar block. However, she continued to sleep well, ambulate and sit with less pain, and require minimal pro re nata (PRN) opioids. The patient agreed to repeat the ganglion impar block, and she had no recurrence of CPP for the following 6 months. The patient was later admitted for cardiopulmonary decompensation secondary to rapid progression of her metastatic disease, and she was discharged to hospice care.

\section{DISCUSSION}

For the past 2 decades, advances in clinical oncology have opened new avenues for cancer treatment (6). The evolution of diagnostic and therapeutic modalities has profoundly improved the survival rate of patients with cancer (6). Prolonged life expectancy in patients with cancer implies an increased prevalence of chronic pain. Hence, the calls for more effective analgesic control are needed to achieve a better quality of life (6).
The management of cancer pain can be challenging to both health care providers and patients, particularly perineal cancer pain. Control of CPP secondary to malignancy is vastly difficult due to the mixed nature of cancer pain and hence it is often refractory to conventional analgesia $(2,3,5,6)$. The pathophysiology of CPP in patients with cancer remains poorly understood. It is likely multifactorial due to perineal defects, neuronal deafferentation from surgery, phantom rectum syndrome from abdominoperineal resection and ostomy creation, peripheral neuropathy from chemotherapy, compression of nerves from tumor progression and invasion, bowel obstruction from tumor infiltration, radiation-induced fibrosis, and idiopathic pain from co-existing diseases $(5,8)$.

The majority of cancer pain can be adequately controlled using the 3-step analgesic ladder proposed by the WHO in $1986(5,6)$. It has been shown to be effective in controlling $90 \%$ of cancer-related pain and more than $75 \%$ in patients with advanced stages of cancer $(5,8)$. The use of WHO Step 3 analgesics are 


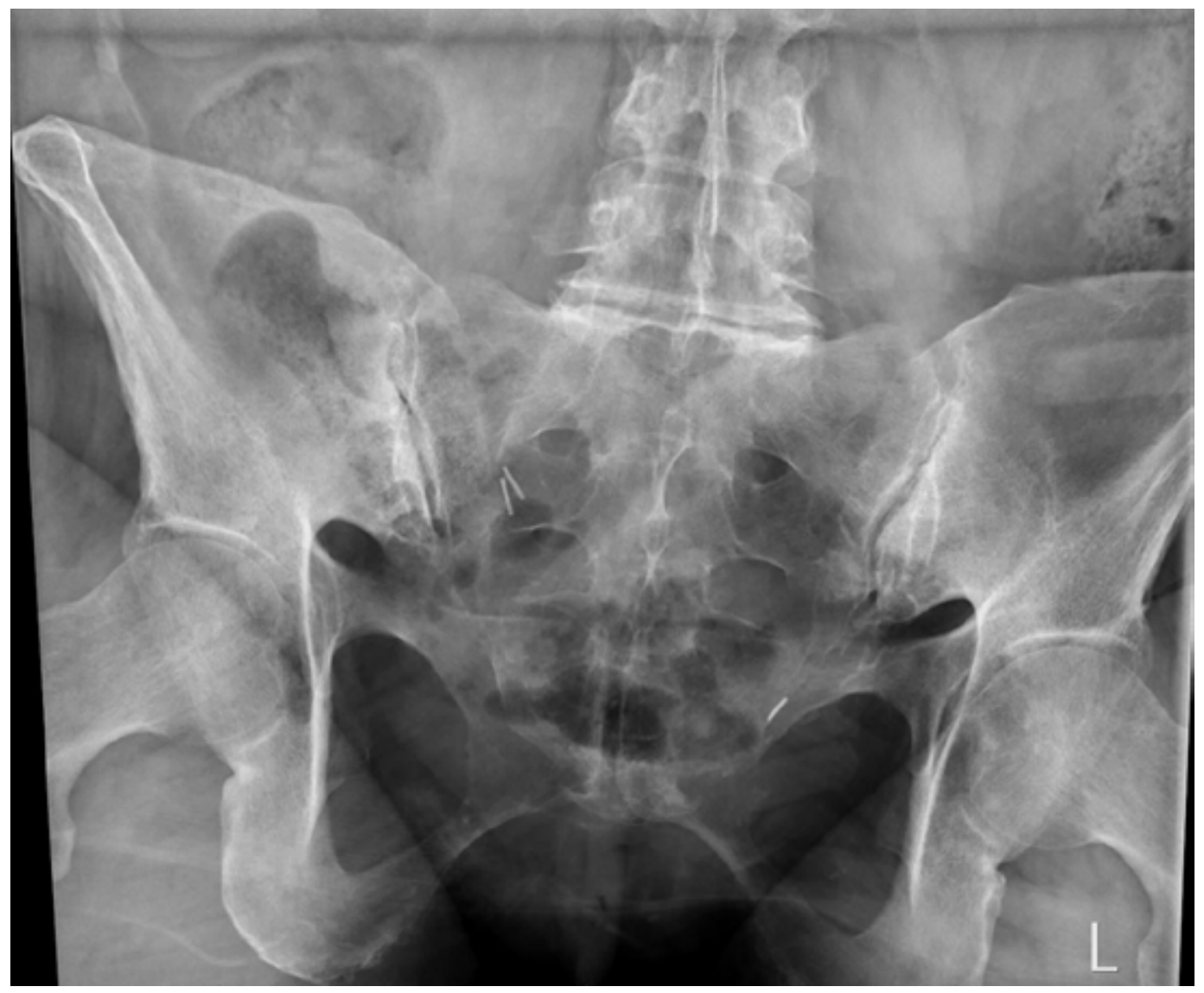

Fig. 2. Anteroposterior radiographic view of the sacrum and coccyx demonstrating an anatomically distorted coccyx with an ill-defined sacrococcygeal junction.

often adequate to relieve cancer pain in the majority of patients, but the side effects of opioids and risks of polypharmacy are highly undesirable (5). Therefore, interventional pain management should be sought early for prompt and effective control of perineal cancer pain.

In our patient, conventional medical management had failed and interventional therapy was sought. The ganglion impar block was challenging due to several anatomical abnormalities. Firstly, our patient had no rectum secondary to APR. Without the rectum as an external landmark, the needle was advanced fluoroscopically into the presacral space with potentially increased risk of hollow viscus perforation. Secondly, the anterior coccyx could not be accessed through the sacrococcygeal ligament due to a distal coccygeal abnormality related to childhood trauma and palliative surgeries, limiting us to proceed with the less desired anococcygeal approach. Thirdly, scarring in the presacral area related to postsurgical changes of APR and radiation therapy made the insertion of a spinal needle and administration of drugs extremely difficult due to significant resistance. Lastly, the patient failed to tolerate being prone due to the presence of an ostomy and significant abdominoperineal pain.

Despite these anatomical challenges, the ganglion impar block was successful. Our patient regained satisfactory functional restoration of daily living. Her 


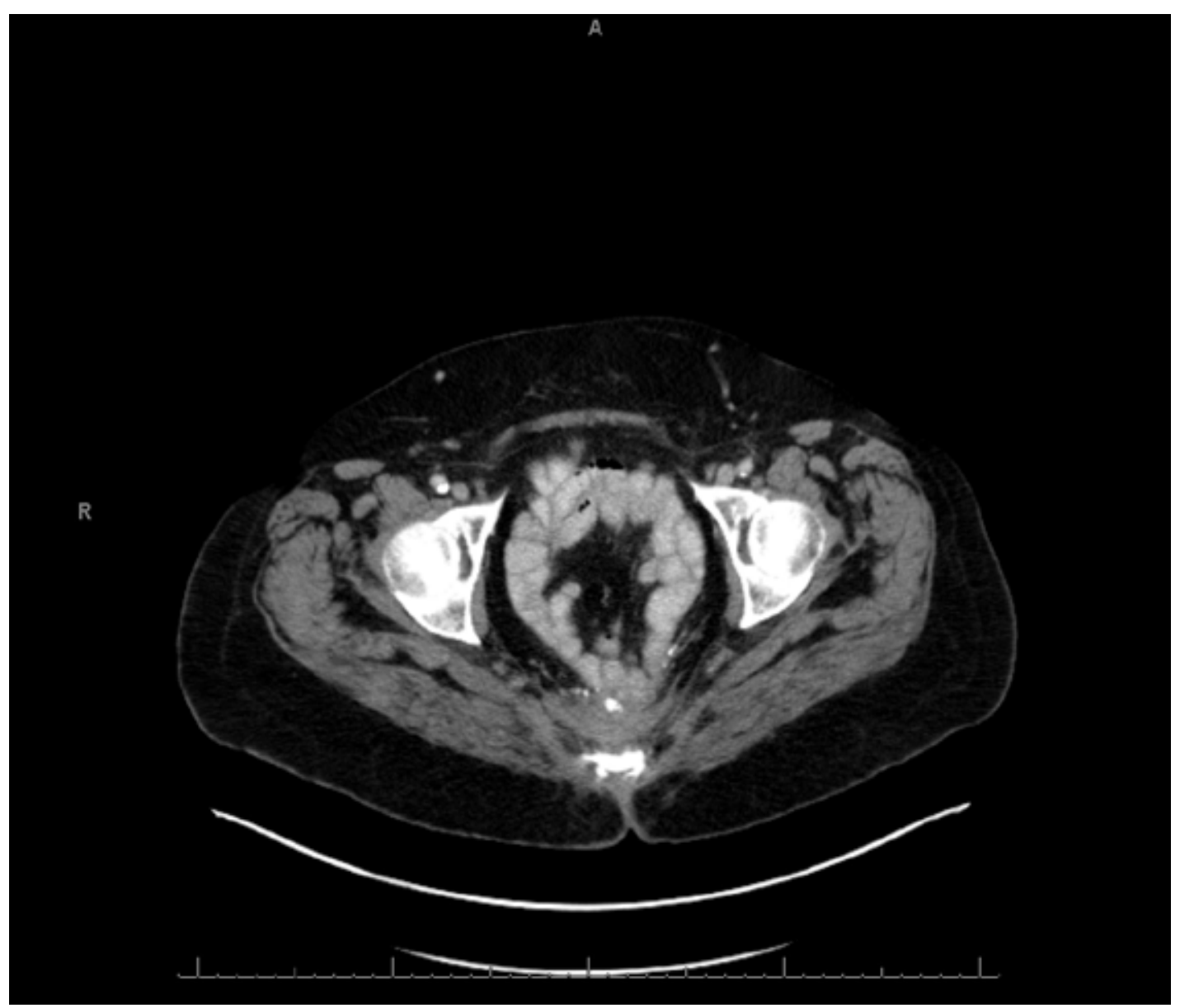

Fig. 3. Computerized tomography scan of the abdomen and pelvis revealing significant scarring within the presacral space.

quality of life improved significantly from palliation of pain, reduced opioid consumption, improved sleep quality and patient satisfaction, increased physical capacity, and better appetite. If her CPP recurs in the future, neurolytic block of the ganglion impar can be considered as a clinically reasonable end point for long-term relief of her pain. Chemical neurolysis using phenol or alcohol for sympathetically mediated perineal pain has been used for a long time and is well-documented in the literature $(3,4,7)$. Although radiofrequency ablation (RFA) of the ganglion impar may also be a good alternative for managing cancerrelated perineal pain, it has only been documented in 3 case reports and a small retrospective study (9-12). Compared to RFA, neurolytic destruction is often more desirable due to the ability of alcohol or phenol to spread locally to reach the ganglion impar, which is commonly located anterior to the coccygeal bone between the first and second coccygeal joints (13). Our patient is not a candidate for RFA as we were not able to access the sacrococcygeal junction due to a significant amount of ossification, narrow joint space, and coccygeal deformity. The literature shows that fluoroscopically guided ganglion impar destruction by RFA has only been conducted via the transsacrococcygeal approach (10-13). Therefore, it may not be technically feasible to perform RFA via the anococcygeal approach due to concerns of the difficulty inserting the RFA probe through the curved spinal needle and the possible shattering of the elec- 


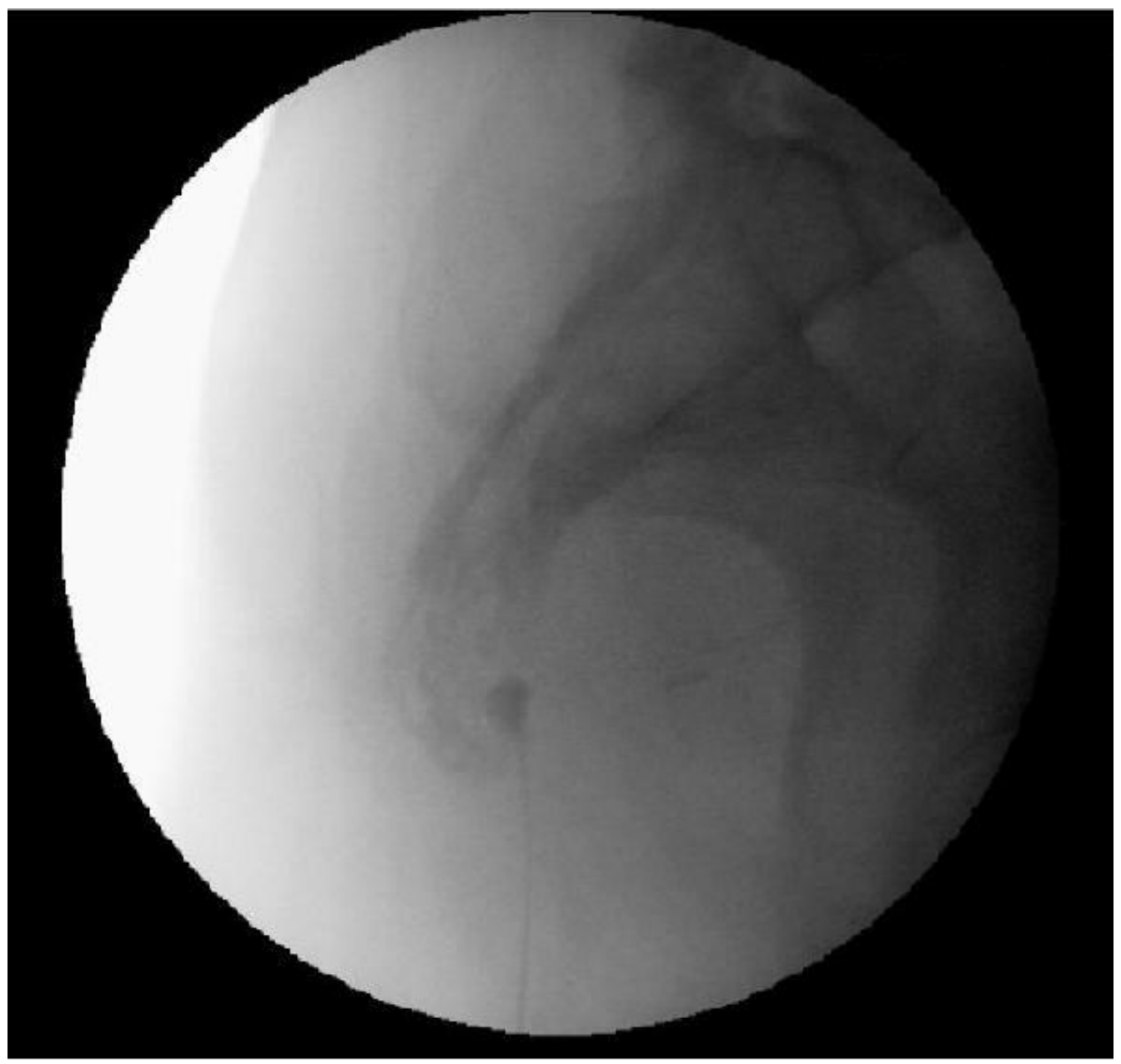

Fig. 4. Lateral radiographic view of the sacrum demonstrating proper placement of the curved spinal needle in the presacral space under fluoroscopic guidance. The coccyx appears to be significantly deformed with the absence of the distal coccygeal segment. The sacrococcygeal segment could not be clearly identified and hence the transsacrococcygeal approach of ganglion impar block was not considered.

trode upon withdrawal from the curved spinal needle.

\section{CONCLUSION}

Ganglion impar block can be used as an effective treatment for CPP in patients with cancer, and, in palliative settings, neurolytic ablation can be considered. It is imperative to realize that a ganglion impar block can be technically challenging in patients with advanced cancer secondary to frequent findings of anatomical abnormalities involving the pelvis, sacrum, coccyx, and perineum, and the inability to tolerate certain positions. Whether or not a ganglion impar block offers definitive therapy for pain relief remains to be established. Further research is warranted on the safety profile and efficacy of ganglion impar block in patients with cancer who have CPP. 
Ganglion Impar Block for Perineal Cancer Pain

\section{REFERENCES}

1. Stowers KH, Hartman AD, Gustin J. Diltiazem for the management of malignancy-associated perineal pain and tenesmus. J Palliat Med 2014; 17: 1075-1077.

2. Lim SJ, Park HJ, Lee SH, Moon DE. Ganglion impar block with botulinum toxin type A for chronic perineal pain - a case report. Korean J Pain 2010; 23: 65-69.

3. Malec-Milewska M, Horosz B, Koleda I, Sękowska A, Kucia H, Kosson D, Jakiel G.. Neurolytic block of ganglion of Walther for the management of chronic pelvic pain. Video Surgery and Other Mini Invasive Techniques 2014; 9:458-462.

4. Toshniwal GR, Dureja GP, Prashanth SM. Transsacrococcygeal approach to ganglion impar block for management of chronic perineal pain: A prospective observational study. Pain Physician 2007; 10: 661-666.

5. Miguel R. Interventional treatment of cancer pain: The fourth step in the World Health Organization analgesic ladder? Cancer Control 2000; 7:149-156.

6. Birthi $P$, Sloan P. Interventional treatment of refractory cancer pain. Cancer J 2013; 19:390-396.

7. Scott-Warren JT, Hill V, Rajasekaran A. Ganglion impar blockade: A review. Curr Pain Headache Rep 2013; 17:306.

8. Rigor BM Sr. Pelvic cancer pain. J Surg Oncol 2000; 75:280-300.

9. Foye PM. Ganglion impar pulsed radiofrequency for coccyx pain. J Pain Symptom Manage 2011; 41: e11-12

10. Gürses, E. Impar ganglion radiofrequency application in successful management of oncologic perineal pain. J Pak Med Assoc 2014; 64:697-699.

11. Demircay E, Kabatas S, Cansever T, Yilmaz C, Tuncay C, Altinors N. Radiofrequency thermocoagulation of ganglion impar in the management of coccydynia: Preliminary results. Turk Neurosurg 2010; 20:328-333.

12. Usta B, Gozdemir M, Sert H, Muslu B, Demircioqlu RI. Fluoroscopically guided ganglion impar block by pulsed radiofrequency for relieving coccydynia. J Pain Symptom Manage 2010; 39:e1-e2.

13. Reig E, Abejon D, del Pozo C, Insausti J, Contreras R. Thermo coagulation of the ganglion impar or ganglion of Walther: Description of a modified approach. Preliminary results in chronic nononcological patients. Pain Practice 2005; 5:103. 
